\title{
Evolution of Wireless Communication Systems towards Autonomously Managed, Cognitive Radio Functionalities
}

\author{
Markus Muck, Sophie Gault, Didier Bourse \\ Motorola Labs \\ 91193 Gif-sur-Yvette, France \\ Markus.Muck@,Motorola.com
}

\author{
Konstantinos Tsagkaris, Panagiotis Demestichas \\ University of Piraeus \\ Athens, Greece \\ ktsagk@unipi.gr
}

\author{
Zachos Boufidis, Makis Stamatelatos, Nancy Alonistioti \\ University of Athens \\ Athens, Greece \\ boufidis@di.uoa.gr
}

\begin{abstract}
This contribution presents key functionalities and design approaches of a distributed system architecture as it is studied in the framework of the European $E^{2} R$ II project [1]. An emphasis is laid on policy based self-governance, distributed reconfiguration concepts and corresponding cognitive support functionalities; this support is necessary to assure context awareness in the equipment in order to facilitate (enable) distributed decision making. The idea is to distribute decision making functionalities among network and user equipment elements in order to i) limit the required calculation complexity on the network and user side for the determination of the optimum resource selection strategy, ii) increase the reactivity of the equipment to any context change minimizing the required amount of human interaction and iii) minimize the signaling overhead by broadcasting generic policy rules applicable to all users instead of targeting a user-by-user based reconfiguration approach. A simple use case illustrates how Game Theoretic tools can be used in order to derive suitable policies and how to perform decisions.
\end{abstract}

Keywords- autonomic networking, cognitive networks, reconfiguration, software defined radio

\section{INTRODUCTION}

$\mathrm{T}$ he success of next generation networks is dependent on the availability of functionality- and feature-rich applications that will be accessible via a variety of network infrastructures and terminals. This creates the need for understanding how to design and deploy heterogeneous system concepts and how to efficiently deal with user adaptation and optimum service provision in such a multistandard, multi-operator context.

A key aspect to be studied within the second phase of the $E^{2} R$ project [1] is the efficient management of network and user equipment reconfiguration in a distributed context. Such an approach is expected to require the move from a centralized or partly distributed architecture to a fully distributed solution. A cognitive support is expected to provide the information necessary such that the equipment entities are able to perform autonomous (policy based) decisions with the goal of efficient self-management, self-optimization, self-protection, selfhealing, etc. [2]. In section II, this paper illustrates the required architectural key building blocks and a suitable system architecture. Section III introduces the Reconfiguration Management Plane (RMP) providing network functionalities required for the distributed decision making. The context information is proposed to be communicated to user equipment via a cognitive support as explained in section IV. In section $\mathrm{V}$, some examples will finally illustrate how Game Theoretic decision making may be performed in the considered context. Section VI gives a conclusion.

\section{STRATEGIES AND POLICIES AS THE KEY TO RECONFIGURATION}

In order to assure an efficient operation of a large distributed wireless system, the presence of several, typically heterogeneous, Radio Access technologies (RATs) within the range of a Mobile Terminal (MT) is considered as illustrated in Fig. 1.
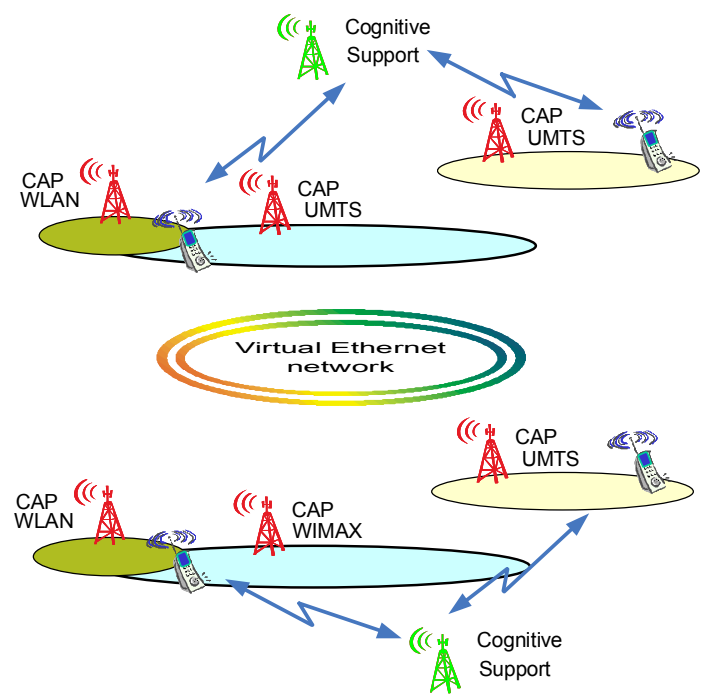

Figure 1: A vision on a distributed system architecture. 


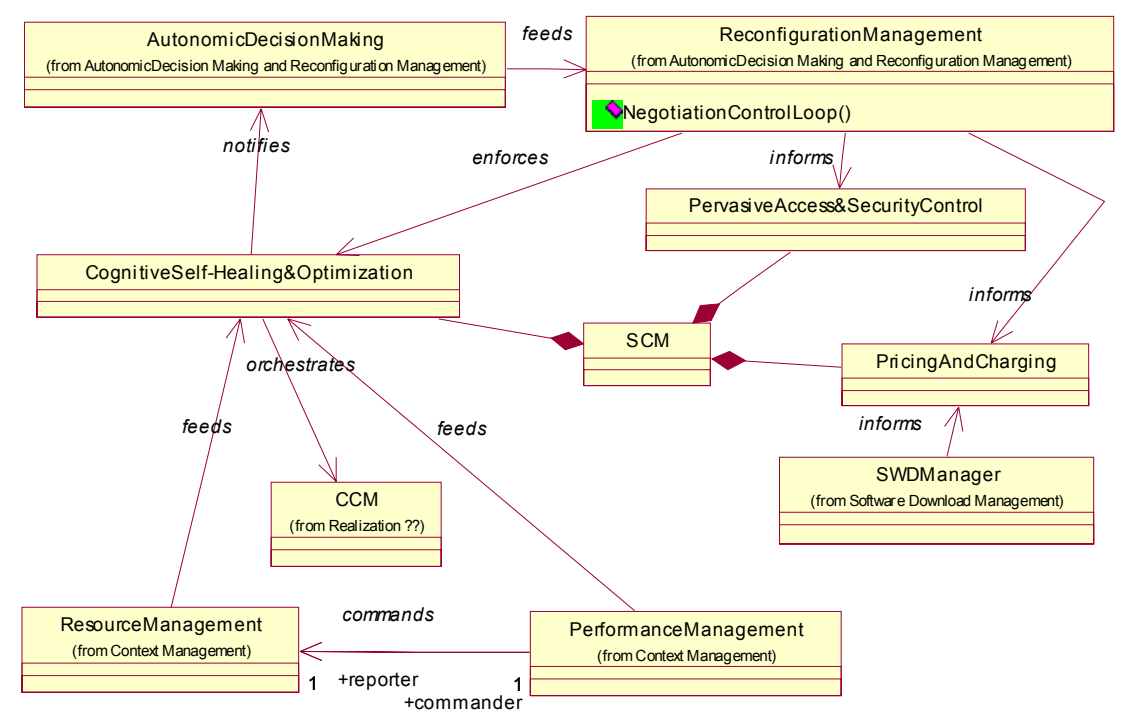

Figure 2. Self-configuration and management capability.

An efficient reconfiguration is assumed to apply autonomous equipment reconfiguration strategies based on global policy provision; this concept allows to react efficiently on a context change (RATs are added/removed/reconfigured: in the example to be presented in section V, a Cellular Access Point (CAP) is removed), avoiding the typical information exchange for an operator based parameterization.

\section{Reconfiguration Management Plane}

In order to facilitate an autonomic operation of network elements and end-user equipment, a hereafter called Reconfiguration Management Plane (RMP) is introduced (Fig. 3 ). Its goal is to encapsulate the additional intelligence needed in order to trigger, negotiate, decide, and coordinate the reconfiguration of the distributed system by providing capabilities for autonomic decision making and management of the reconfiguration process, cognitive service provision, software download, self-configuration and -management, and context management [3].

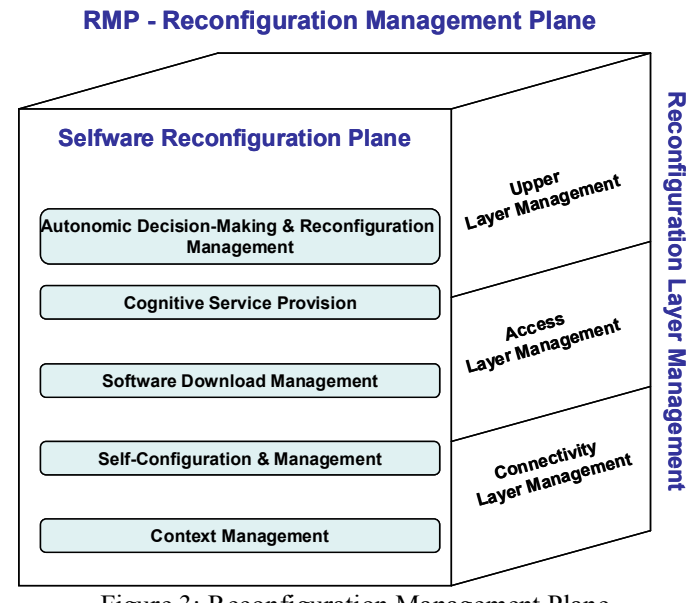

Figure 3: Reconfiguration Management Plane.
Autonomic decision-making and reconfiguration management involves the distributed self-generation of reconfiguration strategies and policy rules on a per equipment level. These policies are not only related to local equipment operations, but could also describe the control of policy authorization in external domains. This would alleviate the need for a set of policy decision points in the network communicating with policy enforcement points on a clientserver basis. In addition, this system capability involves the initiation, maintenance, and tear-down of reconfiguration sessions, whereas guaranteeing end-to-end negotiation of quality of service attributes. Finally, autonomicity requires the capability to contact and communicate with external elements in order to exchange and retrieve contextual information.

Self-configuration and management (see Fig. 2) involves the following key themes:

- Self-optimization refers to the ability to improve the operation of a system based on metrics to be provided by the performance management module and executed by the resource management module. These two modules are integral entities of the context management module.

- Pricing control mechanisms shall be capable of calculating the price for the negotiated resources.

Further details on all functional entities of the RMP can be found in [3].

\section{COGNITIVE SUPPORT}

As already stated, cognitive support is necessary to provide the equipment with context awareness, thus facilitating distributed decision making. Cognitive support is supplied by the network. Specifically, in the network side, the triptych reconfigurability, management and learning is sufficient to designate a network as "cognitive". A cognitive network is able to, reactively or proactively, adapt to the environment requirements and conditions, in principle, by means of selfconfiguration (self-management). Self-configuration is 
applied, for tackling complexity and scalability. The general definition of cognitive networks implies some advanced capabilities in support to the evolution of wireless communication systems towards autonomously managed, cognitive radio functionalities. As part of the selfconfiguration, there are elements (comprising reconfigurable transceivers) that dynamically change the RATs they operate and the spectrum they use, in order to improve capacity and QoS levels. In other words, an element will be changing RATs and/or spectrum, in space and time, in order to adapt to new conditions and requirements. Advanced management functionality to be in charge of finding the best reconfigurations, is then required and deals with "RAT and Spectrum selection and Coverage \& Capacity capabilities discovery" per network element.

The network management functionality to provide a computationally efficient solution to that problem of exploiting the capabilities of candidate reconfigurations is shown in the upper left part of Fig. 4. Capabilities are exploited in the provision of the highest possible QoS levels, at the appropriate capacity levels. This exploitation yields a rating of the candidate reconfigurations, and leads to the selection of the best one.

The input can be classified in three main categories: (i) element capabilities, (ii) demand \& context requirements, and (iii) user profiles. Element capabilities provide information on the candidate configurations of the reconfigurable element. In general, an arbitrary reconfigurable element will have a set of transceivers, each one capable of operating a set of RATs/spectrum. In addition, whenever a reconfigurable transceiver is assigned with a certain configuration (RAT/spectrum), it is also "imbued" with certain capacity and coverage capabilities. Demand \& Context requirements exploit basic monitoring information for estimating traffic and mobility characteristics, which in general comprise the demand and context requirements in the service area. Finally, User Profiles describe the profiles (e.g., preferences, QoS requirements, constraints) of user classes, applications and terminals, as well as the agreements of an operator with other business entities (e.g. other operators, service or software providers).

The above management functionality can be enhanced with the provision of robust (stable and reliable), learning and adaptation, strategies for discovering the potential capabilities (e.g., capacity, coverage) of candidate reconfigurations, in a distributed manner. These capabilities can change over time, as they are influenced by the changing conditions in the environment, especially the behaviour of "near-by" elements.

In any case, at the end of the optimization process the network proposes the best configuration, which corresponds to a certain RAT/spectrum allocation, capacity and coverage capabilities per network element. This info may be then broadcasted to MTs as strategies candidate to be selected. The ultimate goal of the network is to construct such strategies and apply policies in full collaboration with equipment, so as to achieve a satisfactory level of autonomy. Policies (constraints in strategy selection) can be changed via the feedback loop to equipment shown in Fig. 4. An illustrative example is given in the next section.

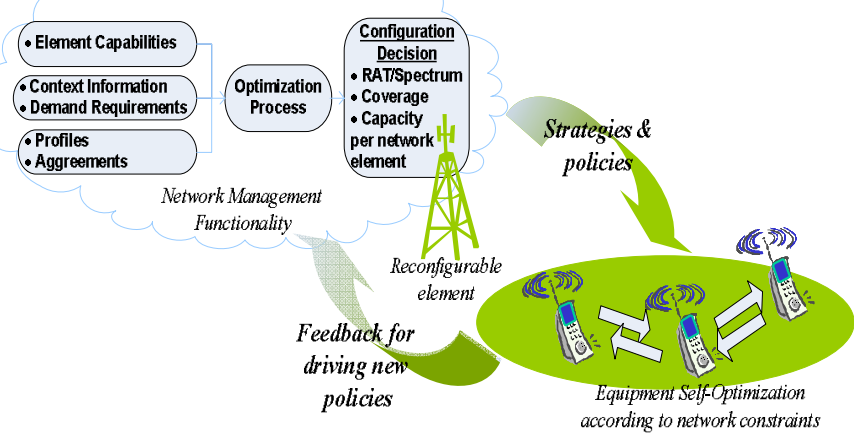

Figure 4. Cognitive network support

\section{EXAMPLE ON GAME THEORETIC DECISION MAKING EXPLOITING THE PRESENCE OF COGNITIVE SUPPORT}

This section illustrates the policy derivation process on the network side and the policy-based self-management concept on the user side by means of an illustrative example: users are able to define autonomously their resource usage strategy within the limits of the constraints imposed by the network.

We choose to consider the simple scenario presented in Fig. 5: two user mobile terminals (MTs) are present in a network which is assumed to be controlled by a single operator. The operator is assumed to switch off one of four available IEEE 802.11n [4,5] Cellular Access Points (CAPs), expecting the user terminals to adapt autonomously to this new context.

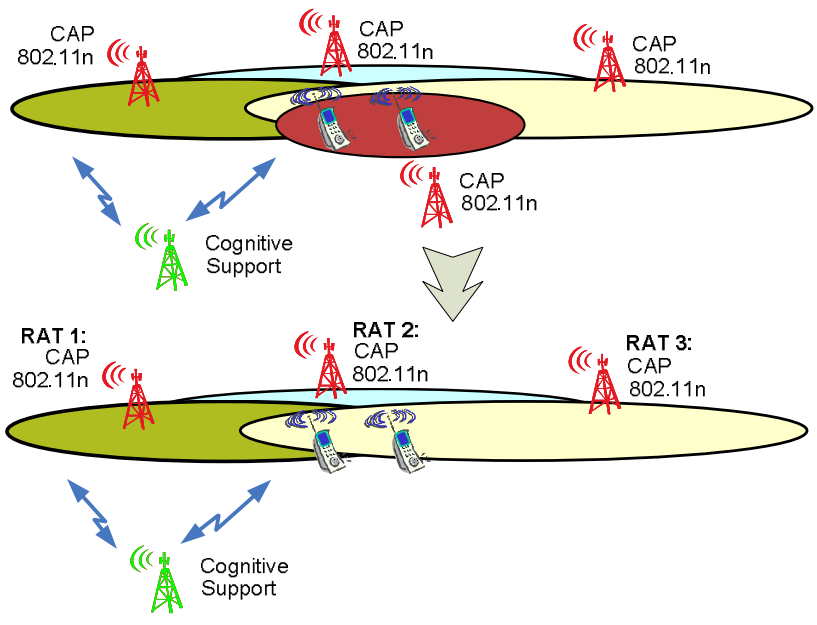

Figure 5: Reconfiguration Example.

The MTs then need to redefine their spectrum / AP usage strategy autonomously. Each MT is assumed to have the choice among seven possible spectrum allocation strategies supplied by the cognitive network support and denoted from S1 to S7: S1: use RAT \#1; S2: use RAT \#2; S3: use RAT \#3; S4: use RATs \#1 and \#2; S5: use RATs \#2 and \#3; use RATs 
\#1 and \#3; S7: use RATs \#1, \#2 and \#3, provided that the MT exists within the coverage area of the elements configured as above. Furthermore, the network configuration imbues each network element with certain throughput (capacity) values.

A simplified throughput computation model is used, assuming a throughput per band (or channel) equal to $D$ bit/s. When a given channel is reserved to only one MT, the total throughput $D$ is available for the MT. In case it is split among two MTs, the total throughput decreases due to collisions: $D$ ' $=D^{*} d$ where $0<d<1$ is a penalty factor, and each MT gets a throughput of $D^{\prime} / 2=D^{*} d / 2$. In the examples below, we choose " $d=0.9$ " for illustration purposes.

The issue addressed is that there are two terminals optimizing their respective resource usage strategies independently, without any knowledge of each others intentions and with subject to their own preferences. The network consequently needs to provide policies (or selection constraints) which ideally force the users to choose a strategy that is both optimum from a global network perspective and from the perspective of each individual user. It is shown in the sequel how to derive suitable policies exploiting Game Theory based tools [6].
The analysis is carried through the $2 D$ game table presented in Tab. 1; the rows and the columns correspond to the strategies of MT1 and MT2 respectively. In addition, the table elements correspond to pairs of throughput values (MT1 throughput, MT2 throughput) normalized by $D$, obtained when MT1 and MT2 are using a given combination of strategies. To give an example: in the first cell on the upper left corner, user 1 chooses "strategy S1" and user 2 equally chooses "strategy S1"; in conclusion, both users are sharing a single channel where collisions may occur and the throughput per user is $1 / 2 * d * D=0.45 * D$. After analyzing the overall game table, the existence of a unique Nash equilibrium when both users choose strategy S7 (red cell) is apparent. In fact, this forms a stable state which no user would find it interesting to deviate from. However, it is not Pareto efficient since better couples of throughputs are obtained with other combinations (yellow cells).

If a given user follows the simple rule of always targeting the maximal throughput, no matter what are the consequences on the other user, he will choose strategy S7 and reach the states corresponding to the green cells.

\begin{tabular}{|c|c|c|c|c|c|c|c|}
\hline & & $\overline{\mathrm{S} 2}$ & $\mathrm{~S} 3$ & $\overline{\mathrm{S} 4}$ & $\Rightarrow$ S5 & S6 & S7 \\
\hline$\overline{S 1}$ & $(0.45,0.45)$ & $(1,1)$ & $(1,1)$ & $(0.45,1.45)$ & $(1,2)$ & $(0.45,1.45)$ & $(0.45,2.45)$ \\
\hline S2 & $(1,1)$ & $(0.45,0.45)$ & $(1,1)$ & $(0.45,1.45)$ & $(0.45,1.45)$ & $(1,2)$ & $(0.45,2.45)$ \\
\hline S3 & $(1,1)$ & $(1,1)$ & $(0.45,0.45)$ & $(1,2)$ & $(0.45, \overline{1.45)}$ & $(0.45,1.45)$ & $(0.45,2.45)$ \\
\hline S4 & $(1.45,0.45)$ & $(1.45,0.45)$ & $(2,1)$ & $(0.9,0.9)$ & $(1.45,1.45)$ & $(1.45,1.45)$ & $(0.9,1.9)$ \\
\hline S5 & $(2,1)$ & $(1.45,0.45)$ & $(1.45,0.45)$ & $(1.45,1.45)$ & $(0.9, \overline{0.9)}$ & $(1.45,1.45)$ & $(0.9,1.9)$ \\
\hline S6 & $(1.45,0.45)$ & $(2,1)$ & $(1.45,0.45)$ & $(1.45,1.45)$ & $(1.45,1.45)$ & $(0.9,0.9)$ & $(0.9,1.9)$ \\
\hline S7 & $(2.45,0.45)$ & $(2.45,0.45)$ & $(2.45,0.45)$ & $(1.9,0.9)$ & $(.9,0.9)$ & $(1.9,0.9)$ & $(1.35,1.35)$ \\
\hline
\end{tabular}

Table 1 - Overall game table ( $1^{\text {st }}$ column: User 1 strategies, $1^{\text {st }}$ line: User 2 strategies $)$.

\begin{tabular}{|c|c|c|c|c|c|c|}
\hline & S1 & S2 & S3 & S4 & S5 & S6 \\
\hline $\mathrm{S} 1$ & $(0.45,0.45$ & $(1,1)$ & $(1,1)$ & $(0.45,1.45)$ & $(1,2)$ & $(0.45,1.45)$ \\
\hline S2 & $(1,1)$ & $(0.45,0.45)$ & $(1,1)$ & $(0.45,1.45)$ & $\begin{array}{l}(0.45 \\
\end{array}$ & $(1,2)$ \\
\hline $\mathrm{S} 3$ & $(1,1)$ & $(1,1)$ & $(0.45,0.45)$ & $(1,2)$ & $(0.45,1.45)$ & $(0.45,1.45)$ \\
\hline S4 & $(1.45,0.45)$ & $(1.45,0.45)$ & $(2,1)$ & $(0.9,0.9)$ & $(1.45,1.45)$ & $(1.45,1.45)$ \\
\hline S5 & $(2,1)$ & $(1.45,0.45)$ & $(1.45,0.45)$ & $(1.45,1.45)$ & $(0.9,0.9)$ & $(1.45,1.45)$ \\
\hline S6 & $(1.45,0.45)$ & $(2,1)$ & $(1.45,0.45)$ & $(1.45,1.45)$ & $(1.45,1.45)$ & $(0.9,0.9)$ \\
\hline
\end{tabular}

Table 2 - Modified game table (1st column: User 1 strategies, 1 st line: User 2 strategies). 
This situation results in an operating point which is suboptimal, in spite of being a Nash equilibrium.

For instance, suppose users play in turn, as represented with the orange arrows on Tab. 1. If users are in an initial state such that both users pick up strategy S1 (the normalized throughput they both achieve equals to $0.45^{*} D$ ) and if user 2 is the first to play, he will try to achieve the maximal throughput and therefore chooses strategy S5 (he achieves throughput equal to $2^{*} D$ instead of $0.45^{*} D$ ). Then given the new strategy of user 2 , user 1 will try to maximize its throughput in turn and chooses strategy S7 (the normalized throughput he achieves equals to $1.9^{*} D$ instead of $D$ ). Finally, user 2 responds by also selecting strategy S7 and the equilibrium is reached, since both users achieves throughput equal to $1.35^{*} D$ and no one can improve its throughput by modifying only its strategy unilaterally.

In the framework of this paper we propose to perform the network policy derivation as follows: the idea is to establish controlled competition so as to get the fairest split of resources and reach the states corresponding to the yellow cells. This is achieved by the use of simple policies propagated by the operator, e.g. "do not use strategy S7'. The operating point search is made on the following suitable where strategy S7 has been removed for both users. If the game is played based on this table and users still follow the simple rule of always seeking for the maximal throughput (no matter what are the consequences on the other user), the states corresponding to the yellow cells will systematically be reached.

Suppose again that users play in turn, following the orange arrows represented on Tab. 2. If users are in the same initial state as previously (both users select strategy S1 and achieve normalized throughput equal to $0.45^{*} D$ ) and if user 2 is the first to play, he will choose strategy S5 (he achieves a maximal throughput equal to $2 * D$ instead of $0.45^{*} D$ ). Then given the new strategy of user 2 , user 1 will try to maximize its throughput in turn and chooses indifferently strategy S4 or S6 to get $1.45^{*} D$ instead of $D$. Since the resulting throughput of user 2 is also maximized (he cannot achieve better throughput than $1.45^{*} D$ ), this new configuration is an equilibrium, which is clearly more efficient than the previous equilibrium where users both picked up strategy S7.

At this point it should be pointed out that the use of a fundamental policy rule expressing a constraint on the strategy selection ("do not use strategy S7") makes it possible to avoid sub-optimal Nash equilibrium. We have therefore achieved our initial goal to force the network into a state that is optimum from both the network side and the specific point of view of each user (trying to optimize its communication parameters independently from other users). Note that this policy applies to all users and thus requires a low amount of signaling overhead compared to a user-by-user configuration approach.

Obviously, the simplicity of the resulting policy definition of this example may not be inherent to realistic scenarios comprising both a large number of users and numerous distinct RATs. In these cases, however, one of the following approaches may be applied: i) assuming that no simple set of policies is available guaranteeing the system convergence to the optimum working points, the upper approach is expects the network to identify the most undesirable cases (i.e. lowest network capacity) and to provide suitable policies avoiding these convergence points. This approach is typically applicable if a large number of (quasi-)optima coexist with a number of highly sub-optimum working points.

ii) assuming that a full analysis of all game table contents is not feasible due to calculation complexity constraints, the network is able to analyze the optimality of the current network configuration and to adapt the policies dynamically if the current working point is undesirable, e.g. from a network capacity point of view.

Note that the upper considerations are generic and applicable to theoretically any heterogeneous RAT configuration. In particular, a scenario comprising unlicensed (WiMAX, WiFi, etc.) and licensed (GSM, 3GPP, etc.) standards can be envisaged.

\section{CONCLUSION}

This paper presents a system concept suitable for a heterogeneous RAT environment. A Reconfiguration Management Plane is introduced for handling the reconfiguration processes in both the network and user equipment. The definition of a cognitive channel furthermore helps user terminals to be context aware and thus enables distributed decision making. The illustrative example of how such a system operates shows that suitable policies for distributed reconfiguration can be derived using Game Theoretic tools. In the given context, a simple rule helps to force the system in a stable and optimum working point.

\section{ACKNOWLEDGMENT}

This work has been performed in the framework of the EU funded project $E^{2} R$ II. The authors would like to acknowledge the contributions of their colleagues from the $E^{2} R$ II consortium.

\section{REFERENCES}

[1] End to End Reconfigurability Phase II (E2RII), IST-2003-507995 E2R, http://www.e2r.motlabs.com

[2] J. Strassner, "Autonomic networking - theory and practice", In Proc. 9th IFIP/IEEE International Symposium on Network Management (IM’2005), Nice, France, May 2005

[3] Z. Boufidis, N. Alonistioti, M. Stamatelatos, D. Bourse, K. El-Khazen, and M. Muck, "Evolution of the Reconfiguration Management Plane for Autonomic Communications", Proc. $15^{\text {th }}$ IST Mobile and Wireless Commun. Summit, Mykonos, Greece, June 2006.

[4] S. Coffey, A. Stephens et al., "Joint Proposal: High throughput extension to the 802.11 Standard: PHY", IEEE 802.11-05/1102r4, IEEE Standards Department, Standard Proposal, Hawaii, USA, January 2006.

[5] S. Coffey, A. Stephens et al., "Joint Proposal: High throughput extension to the 802.11 Standard: MAC", IEEE 802.11-05/1095r4, IEEE Standards Department, Standard Proposal, Hawaii, USA, January 2006.

[6] D. Fudenberg and J. Tirole, "Game Theory", MIT Press, ISBN 0-26206141-4, USA, 1991. 